\title{
Thyroid stimulating hormone suppression time on cardiac function of patients with differentiated thyroid carcinoma
}

\author{
Ruihua Wang ${ }^{1}$, Liu Yang ${ }^{1}$, Shui Jin², Xingmin Han and Baoping Liu*
}

\begin{abstract}
Background: This study was to investigate the influence of thyroid stimulating hormone (TSH) suppression time on the cardiac function of differentiated thyroid carcinoma (DTC) patients.

Methods: 105 DTC patients were divided into strict TSH suppression group (model group, TSH $\leq 0.1 \mathrm{mU} / \mathrm{L}$ ) and general TSH suppression group (control group, TSH $>0.1 \mathrm{mU} / \mathrm{L}$ ). According to the suppression time, these two groups were respectively divided into three groups: group within half a year, group between half a year and a year and group more than a year. Gated myocardial perfusion imaging was applied to observe differences of left ventricle (LV) myocardial perfusion, LV diastolic and systolic function and LV systolic synchrony in every group.

Results: The left ventricular diastolic function, systolic synchrony and myocardial perfusion level of model group decreased with prolonged suppression time. The values of left ventricular EF, PFR and BPM in patients less than half a year were higher than those in 6 months to 1 year for control group.

Conclusion: Thyroid stimulating hormone suppression can influence the cardiac function of patients and with the prolongation of suppression time, regardless of the level of TSH suppression, the possibility of cardiac function depression in patients will increase. TSH may lower the risk of cardiovascular disease in high-risk patients than those in TSH patients with moderate or low risk. The drugs improving cardiac function should be used cooperatively in different suppression period to decrease the occurrence rate of cardiac adverse reactions.
\end{abstract}

Keywords: Thyroid stimulating hormone, Differentiated thyroid carcinoma, Cardiac function, Suppression time, Myocardial perfusion

\section{Background}

Differentiated thyroid carcinoma (DTC) is a common malignant tumor affecting endocrine system and accounts for $90 \%$ of thyroid carcinomas $[1,2]$. Thyroid stimulating hormone (TSH) suppression is a key step for DTC treatment and has been applied wildly in clinical practice. However, long-term TSH suppression may lead to an exogenous subclinical hyperthyroidism and increase thyroxine (T4) level and T4/triiodothyronine

\footnotetext{
*Correspondence: baopingliu_zzu@163.com

1 Department of Nuclear Medicine, The First Affiliated Hospital, College of Medicine, Henan Medical Key Laboratory of Molecular Imaging, Zhengzhou University, 1 Jianshe East Road, Zhengzhou 450052, Henan, China

Full list of author information is available at the end of the article
}

(T3) level [3], which may induce many adverse reactions and negative effects on cardiac function [4-6]. At present, the specific TSH suppression level to the best therapeutic effect is controversial. A research in Japan showed no difference in disease free survival between patients with TSH suppression $<0.1 \mathrm{mU} / \mathrm{L}$ and patients with TSH suppression $>0.1 \mathrm{mU} / \mathrm{L}$. However, other research showed TSH suppression $<0.1 \mathrm{mU} / \mathrm{L}$ could significantly improve the prognostic recovery of patients with high-risk thyroid cancer [7, 8]. In this investigation, gated myocardial perfusion imaging (GMPI) [9] was applied to observe the influence of different TSH suppression levels and TSH suppression time on cardiac function from the perspective of left ventricle (LV) myocardial perfusion, LV diastolic and systolic function and LV systolic synchrony so

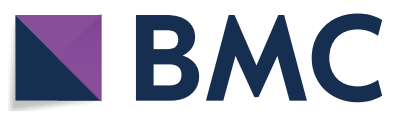

(c) The Author(s) 2018. This article is distributed under the terms of the Creative Commons Attribution 4.0 International License (http://creativecommons.org/licenses/by/4.0/), which permits unrestricted use, distribution, and reproduction in any medium, provided you give appropriate credit to the original author(s) and the source, provide a link to the Creative Commons license, and indicate if changes were made. The Creative Commons Public Domain Dedication waiver (http://creativecommons.org/ publicdomain/zero/1.0/) applies to the data made available in this article, unless otherwise stated. 
as to provide a theoretical basis for the use of different drugs which can improve cardiac function in different treatment period.

\section{Methods \\ Patients}

105 DTC patients in our hospital from October 2014 to January 2016 were collected. This study was approved by the ethic committee of First Affiliated Hospital of Zhengzhou University. All patients had no history of heart disease or related diseases; all patients had not received any medication that might affect heart function. The data were analyzed anonymously. After total thyroidectomy, ${ }^{131}$ I treatment and TSH suppression (L-thyroxine) were performed. Free triiodothyronine (FT3), free thyroxine (FT4) and TSH level in patients' serum were tested every 2 or 3 months (Table 1 ).

According to the standard proposed by the American Thyroid Association [10], patients with highrisk thyroid cancer were recommended a TSH suppression $\leq 0.1 \mathrm{mU} / \mathrm{L}$, and moderate- and lowrisk patients were recommended a TSH suppression $>0.1 \mathrm{mU} / \mathrm{L}$. According to TSH level in serum, all patients were divided into strict TSH suppression group (model group, $\mathrm{TSH} \leq 0.1 \mathrm{mU} / \mathrm{L}, 64$ patients) and general TSH suppression group (control group, $\mathrm{TSH}>0.1 \mathrm{mU} / \mathrm{L}, 41$ patients). In model group, the average age was $46.56 \pm 9.16$, average treatment time was $0.99 \pm 0.71$ (year). In control group, the average age was $45.92 \pm 10.89$, average treatment time was $1.05 \pm 1.09$ (year). There was no statistical difference in both two groups $(P>0.05)$.

According to TSH suppression time (within half a year, between half a year and a year and more than a year), these two groups above were respectively divided into three groups and the influence of suppression time on cardiac function was studied. The model group was divided into group A (within half a year, 5 patients), group $B$ (between half a year and a year, 21 patients) and group $\mathrm{C}$ (more than a year, 28 patients). The control group was

Table 1 Thyroid function test results before and after TSH treatment

\begin{tabular}{lccc}
\hline & Before TSH treatment & After TSH treatment & P \\
\hline TSH (mU/L) & $2.01 \pm 0.08$ & $4.66 \pm 0.93$ & 0.003 \\
T3 (nmol/L) & $1.67 \pm 0.85$ & $2.23 \pm 0.44$ & 0.051 \\
FT3 (pmol/L) & $3.16 \pm 0.94$ & $4.40 \pm 0.84$ & 0.059 \\
T4 (nmol/L) & $67.96 \pm 0.63$ & $71.16 \pm 0.54$ & 0.050 \\
FT4 (pmol/L) & $11.06 \pm 0.41$ & $12.95 \pm 0.33$ & 0.331 \\
\hline
\end{tabular}

TSH thyroid stimulating hormone, T3 triiodothyronine, FT3 free triiodothyronine, T4 thyroxine, FT4 free thyroxine divided into group $\mathrm{D}$ (within half a year, 17 patients), group $\mathrm{E}$ (between half a year and a year, 8 patients) and group $\mathrm{F}$ (more than a year, 16 patients).

\section{Instruments and examination indexes TSH determinator}

Electrochemistry luminescence immunity analyzer (Cobase 411, Roche, Germany). Electrochemical luminescence detection kit was also bought from Roche Company.

\section{GMPI machine}

It was used for the assessment of patients' cardiac function. SPECT (Symbia-T16, Siemens, Germany) was used for scanning. $99 \mathrm{mTc}-\mathrm{MIBI}$ was used, radiochemical purity $>95 \%$. Imaging agent was bought from HTA CO. LTD.

\section{Main determined indexes of cardiac function Myocardial perfusion indexes}

Summed rest score (SRS) and myocardial perfusion target chart.

\section{Cardiac diastolic and systolic functions indexes}

Beats per minute (BMP), LV end-diastolic volume (EDV), LV end-systolic volume (ESV), left ventricular ejection fraction (LVEF), peak ejection rate (PER), peak filling rate (PFR), mean filling rate over the first third of the end-systolic to end diastolic phase (MFR/3), time to peak filling from end-systole (TTPF) and SRS.

\section{Cardiac phase analysis indexes}

Bandwidth, mean angle of LV myocardium systolic, angle standard deviation (STD) of LV myocardium systolic and entropy of LV myocardium systolic.

\section{Statistical analysis}

All statistical analyses were performed using SPSS version 19.0 software. Independent-samples $T$ test was performed for the comparison of cardiac function data in both model group and control group. ANOVA was used for multiple group comparision. One-way analysis of variance was performed for the comparison in every two groups with different suppression time. There was a significant difference at $P<0.05$.

\section{Results}

Cardiac function data comparison decreased with the increase of TSH suppression time in model group (TSH strict suppression group)

Cardiac function data comparison in model group was showed in Table 2. Compared with group B, ejection fraction (EF) value in group $\mathrm{A}$ was lower, the 
Table 2 Cardiac function data comparison in model group with different suppression time

\begin{tabular}{|c|c|c|c|c|c|c|}
\hline & $\begin{array}{l}\text { Group A: within half } \\
\text { a year ( } 15 \text { patients) }\end{array}$ & $\begin{array}{l}\text { Group B: between half a year } \\
\text { and a year ( } 21 \text { patients) }\end{array}$ & $\begin{array}{l}\text { Group C: more } \\
\text { than a year ( } 28 \text { patients) }\end{array}$ & $\mathrm{P}_{\mathrm{AB}}$ & $P_{A C}$ & $P_{B C}$ \\
\hline EF (\%) & $73.01 \pm 5.18$ & $78.66 \pm 5.03$ & $71.39 \pm 8.72$ & 0.049 & 0.274 & 0.002 \\
\hline PER (EDV/s) & $3.97 \pm 0.85$ & $4.23 \pm 0.44$ & $3.23 \pm 0.60$ & 0.438 & 0.001 & 0.000 \\
\hline PFR (EDV/s) & $3.66 \pm 1.04$ & $3.40 \pm 0.81$ & $2.38 \pm 0.82$ & 0.589 & 0.000 & 0.001 \\
\hline MFR/3 (EDV/s) & $1.66 \pm 0.63$ & $1.66 \pm 0.44$ & $1.36 \pm 10.29$ & 0.999 & 0.090 & 0.050 \\
\hline TTPF (ms) & $149.06 \pm 46.41$ & $160.95 \pm 23.33$ & $172.32 \pm 13.75$ & 0.412 & 0.028 & 0.331 \\
\hline BMP & $80.80 \pm 12.09$ & $77.52 \pm 7.37$ & $75.44 \pm 12.50$ & 0.652 & 0.286 & 0.789 \\
\hline Bandwidth & $24.11 \pm 11.92$ & $25.95 \pm 12.59$ & $35.11 \pm 18.37$ & 0.869 & 0.006 & 0.012 \\
\hline Mean angle $\left(^{\circ}\right)$ & $147.52 \pm 9.71$ & $144.13 \pm 10.97$ & $143.15 \pm 17.27$ & 0.751 & 0.590 & 0.968 \\
\hline Angle STD $\left(^{\circ}\right)$ & $8.64 \pm 6.75$ & $8.00 \pm 5.59$ & $11.85 \pm 10.47$ & 0.972 & 0.454 & 0.252 \\
\hline Entropy (\%) & $27.26 \pm 11.86$ & $28.28 \pm 8.88$ & $36.92 \pm 10.30$ & 0.953 & 0.013 & 0.013 \\
\hline SRS & $1.20 \pm 1.42$ & $0.95 \pm 1.32$ & $2.88 \pm 2.00$ & 0.898 & 0.007 & 0.001 \\
\hline $\begin{array}{l}\text { Reduced perfusion } \\
\text { ratio (\%) }\end{array}$ & 16.67 & 33.33 & 67.86 & - & - & - \\
\hline
\end{tabular}

EF ejection fraction, $P E R$ peak ejection rate, EDV end-diastolic volume, $P F R$ peak filling rate, MFR/3 mean filling rate over the first third of the end-systolic to end diastolic phase, TTPF time to peak filling from end-systole, BMP beats per minute, STD standard deviation, SRS summed rest score

difference was statistically significant $(P<0.05)$, there was no notable difference on other cardiac function indexes. Compared with group $\mathrm{C}$, in group $\mathrm{A}, \mathrm{LVPER}$ and LVPFR were higher $(P<0.05)$, TTPF was shorter $(P<0.05)$, bandwidth and entropy of LV myocardium systolic were smaller $(P<0.05)$. Compared with group $C$, in group $B, E F$ value, LVPER, LVPFR and MFR/3 were higher, bandwidth and entropy of LV myocardium systolic were smaller $(P<0.05)$. The perfusion reduction rate increased with the extension of suppression time. All of above showed that in model group, suppression time had influence on cardiac function. The ventricular synchrony was significantly inhibited when suppression time was more than 1 year.

\section{Cardiac function data comparison decreased with the increase of TSH suppression time in control group (TSH general suppression group)}

Cardiac function data comparison in control group was showed in Table 3. There was no notable difference on the data of LV diastolic and systolic function, myocardial perfusion level and myocardial systolic synchrony in group D and group E. Compared with group F, LVEF value, LVPFR, BMP and SRS were higher in group D $(P<0.05)$. There was no notable difference on the data

Table 3 Cardiac function data comparison in control group with different suppression time

\begin{tabular}{|c|c|c|c|c|c|c|}
\hline & $\begin{array}{l}\text { Group D: within half } \\
\text { a year (17 patients) }\end{array}$ & $\begin{array}{l}\text { Group E: between half a year } \\
\text { and a year ( } 8 \text { patients) }\end{array}$ & $\begin{array}{l}\text { Group F: more } \\
\text { than a year ( } 16 \text { patients) }\end{array}$ & $P_{D E}$ & $P_{D F}$ & $P_{E F}$ \\
\hline $\mathrm{EF}(\%)$ & $75.05 \pm 9.60$ & $72.50 \pm 5.63$ & $57.61 \pm 7.75$ & 0.798 & 0.030 & 0.037 \\
\hline PER (EDV/s) & $4.01 \pm 0.86$ & $3.81 \pm 0.36$ & $2.66 \pm 0.82$ & 0.857 & 0.401 & 0.035 \\
\hline PFR (EDV/s) & $3.71 \pm 0.90$ & $3.22 \pm 0.52$ & $2.00 \pm 0.73$ & 0.395 & 0.028 & 0.004 \\
\hline MFR/3 (EDV/s) & $1.36 \pm 0.41$ & $1.68 \pm 0.20$ & $0.55 \pm 0.31$ & 0.138 & 0.246 & 0.006 \\
\hline TTPF (ms) & $156.94 \pm 28.01$ & $153.33 \pm 33.59$ & $117.77 \pm 13.30$ & 0.945 & 0.994 & 0.016 \\
\hline $\mathrm{BMP}$ & $88.17 \pm 7.98$ & $77.15 \pm 7.23$ & $52.97 \pm 13.80$ & 0.098 & 0.001 & 0.010 \\
\hline Bandwidth & $22.82 \pm 17.29$ & $39.94 \pm 29.22$ & $22.89 \pm 8.80$ & 0.087 & 0.423 & 0.019 \\
\hline Mean angle $\left({ }^{\circ}\right)$ & $156.06 \pm 14.21$ & $142.33 \pm 14.27$ & $101.15 \pm 18.30$ & 0.187 & 0.060 & 0.004 \\
\hline Angle STD $\left(^{\circ}\right)$ & $5.40 \pm 6.32$ & $10.63 \pm 10.61$ & $7.02 \pm 6.77$ & 0.291 & 0.786 & 0.544 \\
\hline Entropy (\%) & $22.88 \pm 10.51$ & $33.33 \pm 14.79$ & $21.94 \pm 12.95$ & 0.185 & 0.448 & 0.013 \\
\hline SRS & $0.82 \pm 0.48$ & $0.67 \pm 1.63$ & $1.67 \pm 1.88$ & 0.973 & 0.195 & 0.307 \\
\hline $\begin{array}{l}\text { Reduced perfusion } \\
\quad \text { ratio }(\%)\end{array}$ & 11.76 & 16.67 & 61.11 & - & - & - \\
\hline
\end{tabular}

EF ejection fraction, $P E R$ peak ejection rate, EDV end-diastolic volume, $P F R$ peak filling rate, MFR/3 mean filling rate over the first third of the end-systolic to end diastolic phase, TTPF time to peak filling from end-systole, BMP beats per minute, STD standard deviation, SRS summed rest score 
of LV diastolic and systolic function, myocardial perfusion level and myocardial systolic synchrony in group E and group F. All of above showed that in control group, suppression time had influence on cardiac function. The cardiac function was significantly inhibited when suppression time was more than 1 year, increasing the risk of cardiovascular disease.

\section{Strict suppression of TSH can inhibit cardiac function in patients within suppression time more than a year} Data analysis was performed in group A and group D, group $\mathrm{B}$ and group $\mathrm{E}$, group $\mathrm{C}$ and group $\mathrm{F}$, respectively, so as to further study the different influence on cardiac function with the same suppression time in both model group and control group. As for myocardial perfusion level, the occurrence rate of myocardial perfusion reduction in model group was higher than that in control group in spite of suppression time. SRS difference in the suppression time within half a year and more than a year showed statistical significance $(P<0.05)$. As for other cardiac function, there was no notable difference on systolic and diastolic function and phase analysis in both model group and control group with the same suppression time (within half a year). For the suppression time between half a year and a year, LVEF value and PER were higher in model group than those in control group $(P<0.05)$ (Table 4$)$. When the suppression time was more than a year, LVPER, LVPFR and MFR/3 decreased in both model group and control group, TTPF was also delayed. In model group, the bandwidth and entropy of LV myocardium systolic were higher than that in control group $(P<0.05)$ (Table 5). Cardiac function data comparison in model group and control group before TSH suppression treatment has no difference (Table 6).

\section{Discussion}

Cardiac function data comparison was performed in group A, group B and group $C$. The result shows that LVEF value and systolic function increased at the early stage and decreased at later stage, while LV diastolic function, myocardial systolic synchrony and myocardial perfusion level decreased with the extension of

Table 4 Cardiac function data comparison in group B and group $E$

\begin{tabular}{llll}
\hline & Group B (21 patients) & Group E (6 patients) & P \\
\hline EF (\%) & $78.66 \pm 5.03$ & $72.50 \pm 5.63$ & 0.016 \\
PER (EDV/s) & $4.23 \pm 0.44$ & $3.81 \pm 0.36$ & 0.043 \\
$\begin{array}{l}\text { Reduced } \\
\begin{array}{l}\text { perfusion } \\
\text { ratio (\%) }\end{array}\end{array}$ & 33.33 & 16.67 & - \\
\hline
\end{tabular}

EF ejection fraction, $P E R$ peak ejection rate, EDV end-diastolic volume
Table 5 Cardiac function data comparison in group C and group $F$

\begin{tabular}{llll}
\hline & Group C (28 patients) & Group F (18 patients) & P \\
\hline PER (EDV/s) & $3.23 \pm 0.60$ & $3.66 \pm 0.82$ & 0.046 \\
PFR (EDV/s) & $2.38 \pm 0.82$ & $3.00 \pm 0.73$ & 0.041 \\
MFR/3 (EDV/s) & $1.36 \pm 10.29$ & $1.55 \pm 0.31$ & 0.040 \\
TTPF (ms) & $172.32 \pm 13.75$ & $157.77 \pm 13.30$ & 0.001 \\
Bandwidth & $35.11 \pm 18.37$ & $29.89 \pm 8.80$ & 0.049 \\
Entropy (\%) & $36.92 \pm 10.30$ & $27.94 \pm 12.95$ & 0.012 \\
SRS & $2.88 \pm 2.00$ & $1.67 \pm 1.88$ & 0.046 \\
Reduced & 67.86 & 61.11 & - \\
$\quad \begin{array}{l}\text { perfusion } \\
\text { ratio (\%) }\end{array}$ & & & \\
\hline
\end{tabular}

$P E R$ peak ejection rate, EDV end-diastolic volume, $P F R$ peak filling rate, $M F R / 3$ mean filling rate over the first third of the end-systolic to end diastolic phase, TTPF time to peak filling from end-systole, SRS summed rest score

suppression time. Then cardiac function data comparison was performed in group $D$, group $E$ and group $\mathrm{F}$. The result shows that when suppression time was within half a year, LVEF, LVPFR and BMP were higher than that with the suppression time between half a year and a year, which indicates that except for $\mathrm{TSH}$ suppression, there is still another factor which has influence on cardiac function. However, comparing model group with control group, we can find that TSH suppression is still the main influence factor for DTC patients, especially for patients with long-term suppression. However, strict TSH suppression can inhibit patients' cardiac function, which indicates that high-risk patients with TSH suppression have a higher occurrence rate of cardiovascular disease than moderate- and low-risk patients with TSH suppression.

At the early stage of suppression (within half a year), there was no notable difference on myocardial perfusion level in both control group and model group. When the suppression time was more than a year, notable difference on myocardial perfusion level appeared, and LV myocardial perfusion level decreased in model group. Among patients with suppression time between half a year and a year, LVEF value and PER were higher in model group than that in control group. The possible reason may be that when TSH suppression was performed, high physiological doses of exogenous thyroid hormone were used to improve cardiac load through improving the activity of Ca-ATPase in sarcoplasmic reticulum. At the early stage, it performed as compensatory increase of LV systolic function and the increase of cardiac output function [11]. When the suppression time was more than a year, cardiac reserve capacity was declined. Thus LVPER in model group was lower than that in control group. 
Table 6 Cardiac function data comparison in model group and control group before TSH suppression treatment

\begin{tabular}{llll}
\hline & Model group $(\mathbf{n}=\mathbf{6 4})$ & Control group $(\mathbf{n = 4 1 )}$ & $\boldsymbol{P}$ \\
\hline EF (\%) & $83.01 \pm 5.08$ & $82.66 \pm 5.53$ & 0.073 \\
PER (EDV/s) & $4.67 \pm 0.85$ & $4.23 \pm 0.44$ & 0.051 \\
PFR (EDV/s) & $5.16 \pm 0.94$ & $5.40 \pm 0.84$ & 0.059 \\
MFR/3 (EDV/s) & $1.96 \pm 0.63$ & $2.16 \pm 0.54$ & 0.050 \\
TTPF (ms) & $179.06 \pm 36.41$ & $170.95 \pm 23.33$ & 0.331 \\
BMP & $90.80 \pm 11.29$ & $87.62 \pm 6.37$ & 0.789 \\
Bandwidth & $27.11 \pm 11.42$ & $28.95 \pm 10.49$ & 0.052 \\
Mean angle ( $\left.{ }^{\circ}\right)$ & $201.52 \pm 9.31$ & $203.13 \pm 8.97$ & 0.968 \\
Angle STD $\left(^{\circ}\right)$ & $9.34 \pm 6.01$ & $9.00 \pm 4.19$ & 0.252 \\
Entropy (\%) & $30.26 \pm 10.26$ & $29.28 \pm 8.91$ & 0.013 \\
SRS & $1.10 \pm 0.42$ & $0.99 \pm 0.32$ & 0.001 \\
Reduced & 26.67 & 29.03 & - \\
perfusion & & & \\
ratio (\%) & & & \\
\hline
\end{tabular}

$E F$ ejection fraction, $P E R$ peak ejection rate, EDV end-diastolic volume, $P F R$ peak filling rate, $M F R / 3$ mean filling rate over the first third of the end-systolic to end diastolic phase, TTPF time to peak filling from end-systole, BMP beats per minute, STD standard deviation, SRS summed rest score

The influence of suppression treatment on LV diastolic function is a long-term course. At the early stage of suppression (within half a year), there was no evidence showed any difference on LV diastolic function in both model group and control group. However, when the suppression time was more than a year, PFR, MFR/3 and TTPF all decreased in model group. The same result showed in the comparison of LV phase analysis, when the suppression time was more than a year, systolic synchronicity data in model group (bandwidth and entropy of LV myocardium systolic) were all higher than that in control group. All of these show that long-term strictly TSH suppression (more than a year) has greater influence on LV diastolic function and systolic synchronicity than that with general TSH suppression.

\section{Conclusions}

This investigation shows that with different suppression time, the influence of TSH suppression on cardiac function is different. The concrete difference needs further study. At present, the commonly used L-thyroxine is a main method of TSH suppression for patients with DTC. The positive effects of L-thyroxine are heart rate acceleration, shortening of myocardial refractory period, conduction of electrocardiogram acceleration, and increasing cardiac synchrony. In clinical treatment, drugs which can improve cardiac function should be used in different suppression period, so as to improve the cardiac hemodynamics, stabilize ventricular rate, decrease the occurrence of thromboembolism caused by atrial fibrillation, reduce the rate and seriousness of paroxysmal atrial fibrillation and improve the cardiac systolic and diastolic function [12-14]. For example, the combination of $\beta$-blocker and TSH suppression has effect for the atrial arrhythmia and diastolic dysfunction caused by TSH suppression [15] and reduces the death rate caused by adverse cardiovascular reactions [16-18]. The combination of atenolol [19], digoxin, angiotensin converting enzyme inhibitor and other cardiovascular drugs can effectively reduce the adverse cardiovascular reactions during the suppression process [20]. Some studies have showed that TSH levels were associated in a positive and linear manner with the TC, non-HDL-C and TG levels in euthyroid nondiabetics with newly diagnosed asymptomatic CHD. TSH in the upper limits of normal range might exert adverse effects on the lipid profile and might represent a risk factor for hypercholesterolaemia and hypertriglyceridaemia in the context of CHD. Maintaining TSH in a relative low normal range might be beneficial for lipid profile in euthyroid non-diabetics with newly diagnosed asymptomatic CHD [21]. At present, this study only applies to specific groups of people, and has not yet included dietary factors in the study, so the conclusion can not be extended to other groups; our next study is to expand the sample size of multi-center study.

\section{Abbreviations \\ TSH: thyroid stimulating hormone; DTC: differentiated thyroid carcinoma; LV: left ventricle; T4: thyroxine; T3: triiodothyronine; GMPI: gated myocar- dial perfusion imaging; FT3: free triiodothyronine; FT4: free thyroxine; SRS: summed rest score; BMP: beats per minute; EDV: end-diastolic volume; ESV: end-systolic volume; LVEF: left ventricular ejection fraction; PER: peak ejection rate; PFR: peak filling rate; MFR: mean filling rate; TTPF: time to peak filling from end-systole; STD: standard deviation; EF: ejection fraction; TC: total cholesterol; CHD: coronary heart disease; TG: triglycerides; Non-HDL-C: non-high density lipoprotein cholesterol.}

\section{Authors' contributions}

RW and BL made contributions to the conception and design of the study. LY, $\mathrm{SJ}$ and $\mathrm{XH}$ made contributions to the acquisition of data, analysis and interpretation of data. RW, SJ and XH drafted the article. BL and LY revised the article for important content. All authors read and approved the final manuscript.

\section{Author details}

${ }^{1}$ Department of Nuclear Medicine, The First Affiliated Hospital, College of Medicine, Henan Medical Key Laboratory of Molecular Imaging, Zhengzhou University, 1 Jianshe East Road, Zhengzhou 450052, Henan, China. ${ }^{2}$ Department of Nuclear Medicine, Zhejiang Cancer Hospital, Hangzhou 310011, Zhejiang, China.

\section{Acknowledgements}

Not applicable.

\section{Competing interests}

The authors declare that they have no competing interests.

\section{Availability of data and materials}

All data generated or analysed during this study are included in this published article. 


\section{Consent for publication \\ Not applicable.}

\section{Ethics approval and consent to participate}

The study not only received informed consent from all patients, but also received the support of the ethics committee of the First Affiliated Hospital of Zhengzhou University.

\section{Funding}

Funding information is not applicable.

\section{Publisher's Note}

Springer Nature remains neutral with regard to jurisdictional claims in published maps and institutional affiliations.

Received: 13 July 2018 Accepted: 9 November 2018 Published online: 19 November 2018

\section{References}

1. American Thyroid Association (ATA) Guidelines Taskforce on Thyroid Nodules and Differentiated Thyroid Cancer, Cooper DS, Doherty GM, Haugen BR, Kloos RT, Lee SL, Mandel SJ, Mazzaferri EL, Mclver B, Pacini F, Schlumberger M, Sherman SI, Steward DL, Tuttle RM. Revised American Thyroid Association management guidelines for patients with thyroid nodules and differentiated thyroidCancer. Thyroid. 2009;19:1167-214.

2. Samuels MH, Kolobova I, Smeraglio A, Peters D, Janowsky JS, Schuff KG. The effects of levothyroxine replacement or suppressive therapy on health status, mood, and cognition. J Clin Endocrinol Metab. 2014:99:843-51.

3. Gammage MD, Parle JV, Holder RL, Roberts LM, Hobbs FD, Wilson S, Sheppard MC, Franklyn JA. Association between serum free thyroxine concentration and atrial fibrillation. Arch Intern Med. 2007;167:928-34.

4. Biondi B, Palmieri EA, Lombardi G, Fazio S. Effects of thyroid hormone on cardiac function: the relative importance of heart rate, loading conditions, and myocardial contractility in the regulation of cardiac performance in human hyperthyroidism. J Clin Endocrinol Metab. 2002;87:968-74

5. Fazio S, Palmieri EA, Lombardi G, Biondi B. Effects of thyroid hormone on the cardiovascular system. Recent Prog Horm Res. 2004;59:31-50.

6. Gazdag A, Nagy EV, Erdei A, Bodor M, Berta E, Szabó Z, Jenei Z. Aortic stiffness and left ventricular function in patients with differentiated thyroid cancer. J Endocrinol Invest. 2015;38:133-42.

7. Cooper DS, Specker B, Ho M, Sperling M, Ladenson PW, Ross DS, Ain KB, Bigos ST, Brierley JD, Haugen BR, Klein I, Robbins J, Sherman SI, Taylor T, Maxon HR 3rd. Thyrotropin suppression and disease progression in patients with differentiated thyroid cancer: results from the National Thyroid Cancer Treatment Cooperative Registry. Thyroid Off J Am Thyroid Assoc. 1998:8:737.

8. Sugitani I, Fujimoto Y. Effect of postoperative thyrotropin suppressive therapy on bone mineral density in patients with papillary thyroid carcinoma: a prospective controlled study. Surgery. 2011;150:1250.

9. Chen J, Garcia EV, Bax JJ, Iskandrian AE, Borges-Neto S, Soman P. SPECT myocardial perfusion imaging for the assessment of left ventricular mechanical dyssynchrony. J Nucl Cardiol. 2011;18:685-94.

10. Haugen BR, Sawka AM, Alexander EK, Bible KC, Caturegli P, Doherty GM, Mandel SJ, Morris JC, Nassar A, Pacini F, Schlumberger M, Schuff K, Sherman SI, Somerset H, Sosa JA, Steward DL, Wartofsky L, Williams MD. American thyroid association guidelines on the management of thyroid nodules and differentiated thyroid cancer task force review and recommendation on the proposed renaming of encapsulated follicular variant papillary thyroid carcinoma without invasion to noninvasive follicular thyroid neoplasm with papillary-like nuclear features. Thyroid Off J Am Thyroid Assoc. 2017;27:481-3.
11. Taillard V, Sardinoux M, Oudot C, Fesler P, Rugale C, Raingeard I, Renard E, Ribstein J, du Cailar G. Early detection of isolated left ventricular diastolic dysfunction in high-risk differentiated thyroid carcinoma patients on TSHsuppressive therapy. Clin Endocrinol (Oxf). 2011;75:709-14.

12. Estes NA 3rd, Halperin JL, Calkins H, Ezekowitz MD, Gitman P, Go AS, McNamara RL, Messer JV, Ritchie JL, Romeo SJ, Waldo AL, Wyse DG, American College of Cardiology/American Heart Association Task Force on Performance Measures, Physician Consortium for Performance Improvement (Writing Committee to Develop Clinical Performance Measures for Atrial Fibrillation), Heart Rhythm Society. ACC/AHA/Physician Consortium 2008 clinical performance measures for adults with nonvalvular atrial fibrillation or atrial flutter: a report of the American College of Cardiology/ American Heart Association Task Force on Performance Measures and the Physician Consortium for Performance Improvement (Writing Committee to Develop Clinical Performance Measures for Atrial Fibrillation): developed in collaboration with the Heart Rhythm Society. Circulation. 2008;117:1101-20.

13. Belluzzi F, Sernesi L, Preti P, Salinaro F, Fonte ML, Perlini S. Prevention of recurrent lone atrial fibrillation by the angiotensin-II converting enzyme inhibitor ramipril in normotensive patients. J Am Coll Cardiol. 2009;53:24-9.

14. Radford MJ, Arnold JM, Bennett SJ, Cinquegrani MP, Cleland JG, Havranek EP, Heidenreich PA, Rutherford JD, Spertus JA, Stevenson LW, Goff DC, Grover FL, Malenka DJ, Peterson ED, Redberg RF, American College of Cardiology, American Heart Association Task Force on Clinical Data Standards, American College of Chest Physicians, International Society for Heart and Lung Transplantation, Heart Failure Society of America. ACC/AHA key data elements and definitions for measuring the clinical management and outcomes of patients with chronic heart failure: a report of the American College of Cardiology/American Heart Association Task Force on Clinical Data Standards (Writing Committee to Develop Heart Failure Clinical Data Standards): developed in collaboration with the American College of Chest Physicians and the International Society for Heart and Lung Transplantation: endorsed by the Heart Failure Society of America. Circulation. 2005;112:1888-916.

15. Biondi B, Fazio S, Carella C, Sabatini D, Amato G, Cittadini A, Bellastella A, Lombardi G, Saccà L. Control of adrenergic overactivity by beta-blockade improves the quality of life in patients receiving long term suppressive therapy with levothyroxine. J Clin Endocrinol Metab. 1994;78:1028-33.

16. Haugen BR, Alexander EK, Bible KC, Doherty GM, Mandel SJ, Nikiforov YE, Pacini F, Randolph GW, Sawka AM, Schlumberger M, Schuff KG, Sherman SI, Sosa JA, Steward DL, Tuttle RM, Wartofsky L. 2015 American thyroid association management guidelines for adult patients with thyroid nodules and differentiated thyroid cancer: the american thyroid association guidelines task force on thyroid nodules and differentiated thyroid cancer. Thyroid. 2016;26:1-133.

17. Shargorodsky M, Serov S, Gavish D, Leibovitz E, Harpaz D, Zimlichman R Long-term thyrotropin-suppressive therapy with levothyroxine impairs small and large artery elasticity and increases left ventricular mass in patients with thyroid carcinoma. Thyroid. 2006;16:381-6.

18. Bahn RS, Burch HB, Cooper DS, Garber JR, Greenlee MC, Klein I, Laurberg P, McDougall IR, Montori VM, Rivkees SA, Ross DS, Sosa JA, Stan MN, American Thyroid Association, American Association of Clinical Endocrinologists. Hyperthyroidism and other causes of thyrotoxicosis: management guidelines of the American thyroid association and American association of clinical endocrinologists. Endocr Pract. 2011;17:456-520.

19. Gullu S, Altuntas F, Dincer I, Erol C, Kamel N. Effects of TSH-suppressive therapy on cardiac morphology and function: beneficial effects of the addition of beta-blockade on diastolic dysfunction. Eur J Endocrinol. 2004;150:655-61.

20. Dahl P, Danzi S, Klein I. Thyrotoxic cardiac disease. Curr Heart Failure Rep. 2008;5:170-6.

21. Xing W, Zhao J. TSH in the upper limits of the normal range is associated with an adverse lipid profile in euthyroid non-diabetics with newly diagnosed asymptomatic coronary heart disease. Heart. 2012;98:E161-2. 